\title{
Perpetual Smoking Compromise the Osmotic Fragility of RBC
}

\author{
Mazhar Mushtaq ${ }^{*}$, Uzma Jabbar, Mumtaz Begum, Naim Ahmad Nazami \\ Biochemistry Department, Shalamar Medical and Dental College, Affiliated with University of Health Sciences, Lahore, Pakistan
}

Email address:

dr_hcg@yahoo.com (M. Musthaq), uzma.jabbar@sihs.org.pk (U. Jabbar), mumtaz.begum@sihs.org.pk (M. Begum), naim.ahmad@sihs.org.pk (N. A. Nazami)

${ }^{*}$ Corresponding author

\section{To cite this article:}

Mazhar Mushtaq, Uzma Jabbar, Mumtaz Begum, Naim Ahmad Nazami. Perpetual Smoking Compromise the Osmotic Fragility of RBC. Biomedical Sciences. Vol. 3, No. 3, 2017, pp. 58-62. doi: 10.11648/j.bs.20170303.11

Received: May 16, 2017; Accepted: June 14, 2017; Published: July 21, 2017

\begin{abstract}
Hydrogen Peroxide (HP) is one of the Reactive Oxygen Species (ROS) causing cellular injury. The present study is aimed to assess the level of HP, catalase, total glutathione (T-GSH) in chronic smokers and compare these with acute smoker, passive smokers and non-smokers. In the peripheral blood of chronic smoker, acute smoker, passive smokers and control subjects, oxidative stress was measured in terms of HP present in the serum; antioxidant status was measured by blood T-GSH and catalase activity. Adverse effects of HP is measured in term of osmotic fragility of RBC. Increase amount of HP in serum of smoker was observed, whereas two fold increase of the HP was detected in the serum of acute smoker and moderate amount was detected in passive smoker as compare to non-smoker. Variability in the measurement of catalase and T-GSH were also significantly evident in the groups. Increase concentration of HP in acute smoker suggested that ROS produced in smoking not only have their adverse effects in the lungs but they can cross the alveolar epithelium. Osmotic fragility test indicated the increase in fragility of RBC membrane of smoker when they are subjected to high concentration of HP in vitro.
\end{abstract}

Keywords: Hydrogen Peroxide, Reactive Oxygen Species, Oxidative Stress, Catalase, Glutathione, Smoker

\section{Introduction}

HP pale-blue covalent liquid, freely miscible with water and apparently able to cross cell membranes readily; makes it ubiquitous molecule and is widely regarded as a serious oxidative stressful agent. Numbers of stressful oxidative conditions do contribute to etiology of different diseases. For healthy aerobic organisms, production of ROS is balanced by the antioxidant defense systems. The balance is not always perfect. Damages molecules have to be repaired e.g. DNA; or replaced e.g. most oxidized proteins. Damage molecule impairs the normal functions of cells and eventually damages the tissue.

Ample data is available in the literature describing HP as being cytotoxic to a wide range of cells. Body exhale it, excrete it and take it in with diet. However, levels of HP at or below 20-50 $\mu \mathrm{M}$ seem to have limited cytotoxicity to many cell types. There is a growing literature showing that HP is used as inter and intra-cellular signaling molecule [1]. Hence these may be a good reason not to eliminate all the HP generated in vivo as it is used in physiological signaling mechanisms. At sites of inflammation, HP generated by activated phagocytes appears to modulate the inflammatory process. Controlling cell proliferation or apoptosis and modulating platelet aggregation [2].

ROS play an important role in the modulation of airway inflammation. There is an evidence of oxidant-antioxidant imbalance in asthma. Amounts of exhaled HP appear greater in subjects with inflammatory lung diseases [3,4] and in cigarette smokers $[5,6]$. Cigarette smoke has been calculated to contain $10^{17}$ oxidant molecules/puff, of which $10^{14}$ are ROS. The gas phase of cigarette smoke mainly contains short lived ROS, such as superoxide radical and nitrogen oxide, both of which immediately react to form highly reactive peroxynitrite. In contrast, the tar phase contains the long lived hydroquinones that undergo redox-cycle to form superoxide radical and hydrogen peroxide via semiquinones, thereby resulting in persistent oxidative stress [7]. Hydroquinones and hydrogen peroxide can enter cells and may even reach the nucleus, where they may cause oxidative DNA damage [8]. 
The evidence for the significant role of ROS and free radicals in cigarette smoke toxicology has been overwhelming in the last decades. Substantial amount of free radicals are introduced exogenously and produced endogenously $[5,6]$. Hydroxyl radical generated by aqueous cigarette tar can cause oxidative damage. Cigarette smoke, respirable fibres and dusts act synergistically in the increasing production of damaging hydroxyl radicals. Cell death induced by cigarette smoke exposure can largely be accounted for an enhancement in oxidative stress [9].

It is well documented fact that cigarette smoke is a potent source of oxidative stress that depletes oxidant in vitro, it is unclear whether it has a similar effect in vivo, particularly in humans. However smokers do have increased dietary requirements of antioxidants to manage oxidative stress [10].

RBCs are highly susceptible to oxidative damage due to the high cell concentration of oxygen and hemoglobin, a powerful promoter of the oxidative process [11]. Hence in vitro, RBCs are a reliable model for the study of oxidative stress [12]. The present study also focuses on RBC to demonstrate the stressful effects of HP which can be measures in term of osmotic fragility.

\section{Methodology}

Cross sectional comparative study. It was conducted at the Biochemistry Department of Shalamar Medical and Dental College, between November 2014 and March 2015. Quantified data obtained from the measurements were compared among the groups. Studied individual were inducted and grouped on the following bases.

\subsection{Exclusion Criteria}

1. Smoker if having diseases like HTN, DM or Lung Pathology is excluded from study.

2. Individual on chronic use of, Phenobarbital, Tranquilizers, Alcohol, Opium or other addictive drugs is excluded from study.

\subsection{Inclusion Criteria}

Group 1-Chronic Smoker is defined as person smoking at-least 20-25 cigarettes a day.

Group 2- Acute Smoker giving the blood sample 5 minutes after smoking one cigarette.

Group 3- Passive Smoker is defined as person inhabiting with smokers for 5-6 hours.

Group 4- Non Smoker is defined as person who does not have any chronic illness, does not smoke and neither he inhabitate with smokers.

\subsection{Blood Collection}

Blood sample was collected, after consent from all individual, Irrespective of the socioeconomic status, marital status and the brand they use. Each of the individual filled the proforma and its contents were explained to them in native language. Phlebotomy method was employed to take blood sample from smoker. Collected samples were labeled and split in to two parts; (i) tube containing EDTA for osmotic fragility test (ii) tube containing clotting agent - serum was separated from this tube and stored for further use. All the stored sample were thawed in one go and used for the measurement of HP, catalase activity and measurements of glutathione. Essentially the same procedure was followed for collection of the blood sample from other groups in the study.

\subsection{Osmotic Fragility Assay}

RBC osmotic fragility was determined according to the method previously described. However slight modifications were made to suite the need of experiment. A total of $0.2 \mathrm{ml}$ of fresh blood was added to falcon tube containing, $0.45 \%$ of $5 \mathrm{ml}$ sodium chloride solution. This concentration of sodium chloride has been well documented in research literature at which hemolysis is initiated. After adding the fresh blood, appropriate amount of HP was also added, so that final concentration of HP in the tube was $100 \mu \mathrm{M}$. It was gently mixed and incubated at room temperature for 30 minutes. Images were taken after gentle inversion of the tubes. After 30 minutes the mixtures were then centrifuged for 2,000 RPM for 5 minutes. Images were taken after the centrifugation. Supernatant was discarded and the amount of precipitated $\mathrm{RBC}$ were measured with micropipette and plotted on graph. The results were expressed as amount of left over RBC after the hemolysis.

\subsection{Measurements}

For the quantification catalase activity and HP; measuring kit Cellbiolab - Cat \# STA-341. Oxiselect was used. Necessarily protocol suggested on the leaflet by the kit manufacturer was followed. All the samples were tested in one batch using appropriate standard provided by the kit manufacturer. Briefly measurements of catalase take place in the following two steps;

$$
\text { Reaction 1: } 2 \mathrm{H}_{2} \mathrm{O}_{2} \stackrel{\text { CATALASE }}{\longrightarrow} 2 \mathrm{H}_{2} \mathrm{O}_{2} \text { (Left over) }+\mathrm{O}_{2}
$$

Left over $\mathrm{H}_{2} \mathrm{O}_{2}$ from the reaction 1 conjunct the chromogenic reagent $\mathrm{A}$ and $\mathrm{B}$ in the presence of HRP catalyst, thus producing measurable product at $520 \mathrm{~nm}$. (Reaction 2 ).

$$
\text { Reaction 2: } \begin{aligned}
& 2 \mathrm{H}_{2} \mathrm{O}_{2 \text { (Left over) }}+\text { DHBS (B) }+ \text { AAP (A) } \\
& \stackrel{\text { HRP }}{\longrightarrow} \text { Quinoneimine Dye } \\
& \text { (mcasurcd at 520) }
\end{aligned}
$$

For the measurement of HP, immediately after the separation of serum $50 \mu \mathrm{L}$ of catalase quencher was added to $100 \mu \mathrm{L}$ of serum and the reaction 2 was repeated.

For the quantification T-GSH, measuring kit - Cellbiolab Cat \# STA-312. Oxiselect was used. Necessarily protocol suggested on the leaflet by the kit manufacturer was followed. All the samples were tested in one batch using appropriate standard provided by the kit manufacturer. 


\subsection{Statistical Analysis}

The data was entered and processed using SPSS 16.0. It was expressed as mean $\pm \mathrm{SD}$. For comparison of means of variables of acute and chronic smoker independent $t$. test is used. For comparison of means of variables of chronic, passive and nonsmoker ANOVA is applied. $P$ values $<0.05$ were regarded as statistically significant.

\section{Results}

Total of 152 individual were inducted and grouped in four grouped depending upon the inclusion criteria as discussed in the methodology. In the serum of inducted individual increase concentration of HP is observed in the serum of chronic smoker,

When compare with non-smoker and passive smoker; Table 1. Two fold increase of HP was present in the sample of acute smoker when compared with chronic smoker; Table 2. No increase of HP was observed in passive smokers when compared with non-smoker.

Catalase is antioxidant metalloid-enzymes, ubiquitous among aerobic organisms, which promote the conversion of HP to water and molecular oxygen. Remarkably decrease concentrations were detected in the chronic smoker individuals of our study, indicating their increase consumption in managing the oxidative stress. No changes in the concentration were noticed in the passive individual and in the samples of acute smoker; indicating that catalase may not be the first line of defense against the rise in HP, Table 1 and 2.

Due to the rapid nature of the reduction of GSSG relative to its synthesis or secretion, the ratio of GSH to GSSG is a good indicator of oxidative stress within cells. T-GSH or GSSG levels can be determined by HPLC or biochemically in microplates. Using spectrophotometric assay kit, it was found that there is decrease in level of T-GSH in the serum of acute smoker as compared to chronic smoker, Table 2. Remarkably no changes in the concentration T-GSH level were observed in the chronic and passive smokers when compared with nonsmoker, Table 1.

Table 1. Comparison of HP, Catalase and T-GSH in chronic, passive and nonsmokers.

\begin{tabular}{lllll}
\hline Variables & $\begin{array}{l}\text { Chronic } \\
\text { Smoker } \\
(\mathbf{N = 3 8 )}\end{array}$ & $\begin{array}{l}\text { Passive } \\
\text { Smoker } \\
(\mathbf{N}=\mathbf{3 8})\end{array}$ & $\begin{array}{l}\text { Non } \\
\text { Smoker } \\
(\mathbf{N}=\mathbf{3 8})\end{array}$ & $\begin{array}{l}\text { ANOVA } \\
\boldsymbol{P} \text { Value }\end{array}$ \\
\hline $\mathrm{HP}-\mu \mathrm{M}$ & $29 \pm 3.1$ & $19 \pm 2.2$ & $18 \pm 1.7$ & $<0.001$ \\
Catalase $-\mathrm{U} / \mathrm{ml}$ & $16 \pm 2.9$ & $35 \pm 3.2$ & $37 \pm 3.5$ & $<0.001$ \\
T-GSH $-\mu \mathrm{M}$ & $29 \pm 3.1$ & $33 \pm 2.2$ & $39 \pm 3.6$ & $<0.001$ \\
\hline
\end{tabular}

Table 2. Comparison of HP, Catalase and T-GSH in chronic and acute smokers.

\begin{tabular}{llll}
\hline Variables & $\begin{array}{l}\text { Chronic } \\
\text { Smoker } \\
(\mathbf{N}=\mathbf{3 8})\end{array}$ & $\begin{array}{l}\text { Acute } \\
\text { Smoker } \\
(\mathbf{N}=\mathbf{3 8})\end{array}$ & $\begin{array}{l}\text { Independent } \text { t. } \\
\text { Test }-\boldsymbol{P} \text { Value }\end{array}$ \\
\hline $\mathrm{HP}-\mu \mathrm{M}$ & $29 \pm 3.1$ & $54 \pm 4.7$ & $<0.001$ \\
Catalase $-\mathrm{U} / \mathrm{ml}$ & $16 \pm 2.9$ & $36 \pm 3.7$ & $<0.001$ \\
$\mathrm{~T}-\mathrm{GSH}-\mu \mathrm{M}$ & $29 \pm 3.1$ & $16 \pm 2.1$ & $<0.001$ \\
\hline
\end{tabular}

To demonstrate the adverse effect of HP on living human cell; RBCs were selected. RBCs are susceptible to attacks by ROS because of their high polyunsaturated fatty acid content and their abundance of iron-rich hemoglobin. Osmotic fragility test is performed in the presence or absence of HP of chronic smoker's blood. Figure 1. Blood of chronic smokers, when incubated with $100 \mu \mathrm{M}$ of HP resulted in hemolysis and very few micro liters of $\mathrm{RBC}$ were precipitated after centrifugation in the tube having HP; Figure 2.

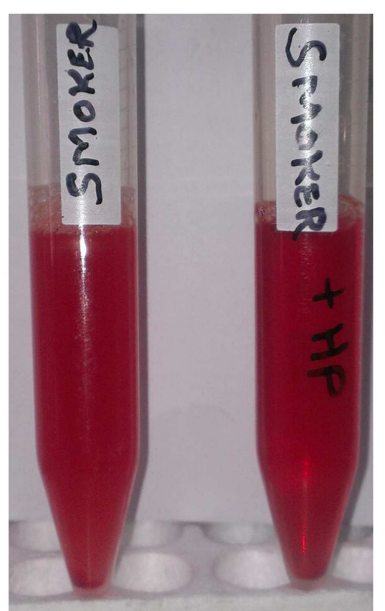

Before centrifugation

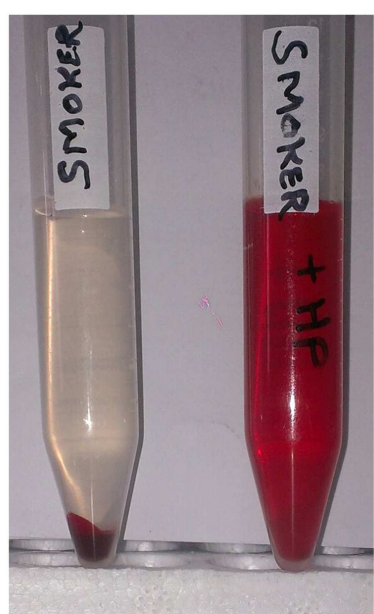

After centrifugation
Figure 1. Osmotic fragility of $R B C$ was assessed by incubating the RBC of chronic smoker with $100 \mathrm{uM}$ of $\mathrm{HP}$ in $0.45 \%$ of $\mathrm{NaCl}$. After 30 minute of incubation, tubes were centrifuge. Hemolysis was seen in the tube containing $H P$, whereas intact RBC were settled down in the tube, making its top clear.

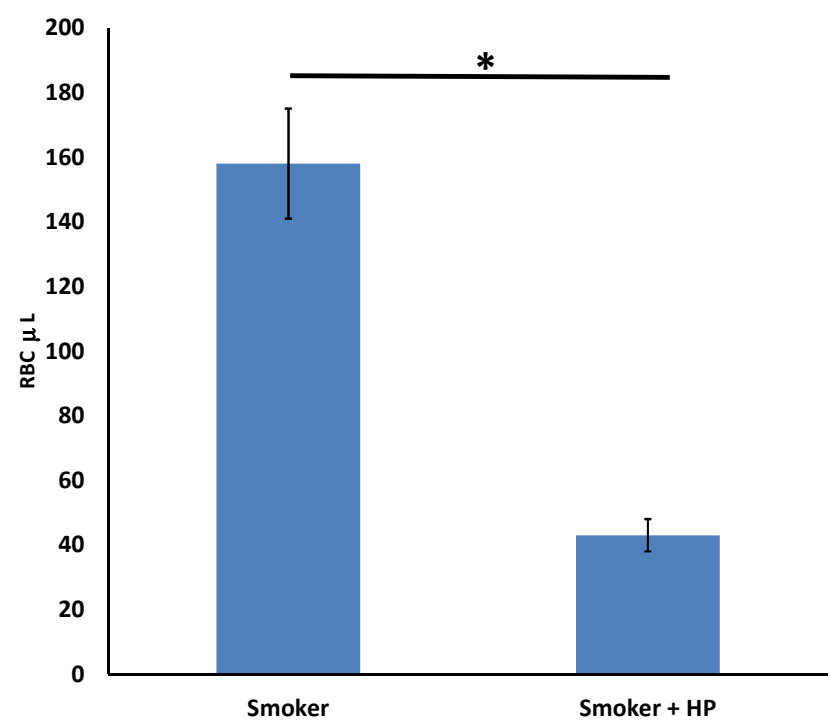

Figure 2. The bar graph represents the mean values of precipitated $R B C$, after centrifugation with $+/-S D$ of three independent experiment of the chronic smoker blood. ${ }^{*} P<0.001$, measured by t test.

\section{Discussion}

Numerous pathological conditions have an oxidative stress component. Overproduction of ROS have been implicated in more than sixty different health conditions and to the etiology of degenerative diseases some of which are 
summarized in Figure 3. However, reactive oxygen species can be beneficial, as they are used by the immune system as a way to attack and kill pathogens, but the type of ROS that actually kill bacteria is still debated [13].

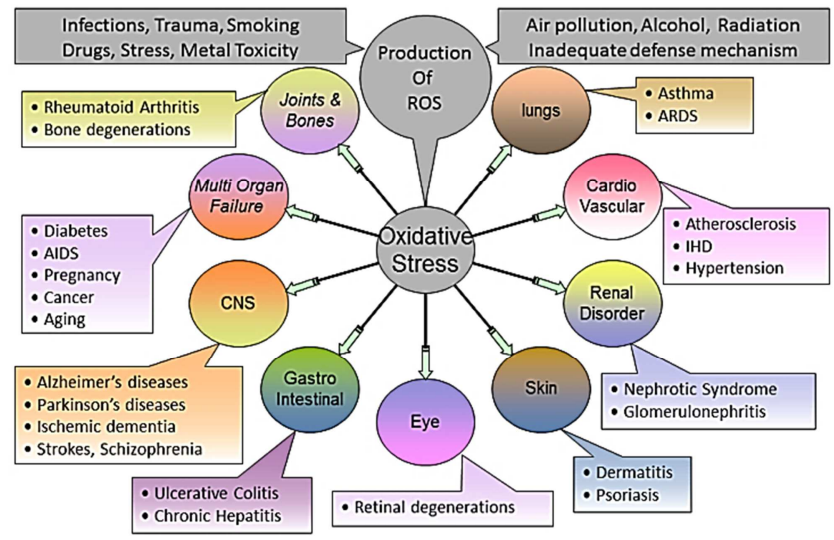

Figure 3. Different factors contributing in the production of ROS and its deleterious effects. Source: Google search from different articles.

Airway epithelia are able to generate ROS upon their exposure to smoke tar. Following cigarette smoking, alterations in alveolar epithelial cells induce an increase in epithelial permeability, a decrease in surfactant production and inappropriate production of inflammatory cytokines [14]. However, the most deleterious effect of cigarette smoke on alveolar epithelial cells is cell death, i.e., either apoptosis or necrosis depending on the magnitude of cigarette smoke exposure. Hence the exposure to smoke tar result in epithelial damage and alter its permeability, thus allowing the movements of HP from alveoli to the blood stream. Increase concentration of HP has been demonstrated in other conditions such as; in the serum of hypertensive patients of different gender and of different ethnicity, in the freshly voided human urine, expired breath of cigarette smokers and increased exhalation of hydrogen peroxide in healthy subjects following cigarette smoke [4-6]. Same impression of increase is perceived in our study, where chronic smoker had significant increase in HP level and on top of it acute smoker has twofold increase in the level of HP. Similarly an amount of HP is greater in patients with inflammatory lung diseases, patient with asthma [3]. Furthermore perpetual exposure of cigarette smoke would elevate the HP level which can create critical imbalance in oxidative and anti-oxidative status of the body. Hence decreases catalase level and T-GSH level in the serum of chronic smoker was observed in our study in the chronic smoker. Similarly bronchiolar epithelial catalase is diminished in smokers [15]. Significantly lower levels of catalase were reported in plasma of diabetic patients. Furthermore low level of catalase may also promote the likelihood of developing type 2 diabetes [16]. Hence the capacity of antioxidant defense systems to catch ROS is highly important in protecting the tissues from oxidative damage caused by endogenous or exogenous ROS.

In addition to enzymatic defenses, there are non-enzymatic defenses against oxidative stress in the form of several metabolites which are important scavengers of ROS. GSH, is one such reductant, which serves many biological functions including maintenance of membrane integrity and protection against free radicals. Reduced glutathione $(\mathrm{GSH})$ is regenerated from its oxidized form (GSSG) by the action of an NADPH dependent reductase (Reaction 3).

\section{Reaction 3: GSSG $+\mathrm{NADPH}+\mathrm{H}+\rightarrow 2 \mathrm{GSH}+\mathrm{NADP}^{+}$}

Acutely, initial decrease of T-GSH had earlier been reported in the smoker [17] this notion is consistent with our study where sharp dip of T-GSH is noted in the acute smoker and somewhat less decrease is observed in the level of TGSH in the chronic smoker. However perpetual smoking may deplete the level of T-GSH thus over-riding of the oxidative stress which can prevails to the chronic inflammation in the lower respiratory-tract epithelium and contribute to the pathogenesis of obstructive lung diseases $[14,17]$. Hence continuously decreased serum antioxidant status or continuously increase oxidative status can catastrophically damage to the epithelial lining of lungs and allowing the movements of HP in the blood which can jeopardize the wellbeing of erythrocyte. In other study it was well demonstrated that RBC membrane of rats can't sustain the oxidative stress of streptozotocin-induced diabetes, however in the same study it was demonstrated that protective agent (antioxidants) when administer in adequate dose enables the RBC to sustain the oxidative stress of diabetes [18-20]. Cigarettes smoking has reached to alarming situation in all societies, irrespective of the gender and age. It was concluded yet in another study that smoking deplete the serum oxidant status level in university student [21]. In other study it was demonstrated that chronic cigarette smoking may induce alternation in membrane permeability resulting in outflow of electrolyte [22]. Deleterious effects of smoking at the molecular level have also been concluded in another study where it is demonstrated that Macular degenerations through specific signaling pathway is evident due to smoking [23]. Yet in another study it is concluded that cigarette smoke condensate on oxidative stress and result in apoptotic cell death through cytochrome P450 pathway [24].

\section{Conclusion}

Finding of this study concludes that glutathione and catalase provide overlapping defenses for the detoxification mechanism of HP. Uneven distribution of oxidant enzymes and highly variable metabolic load of various parts of the mitochondrial network varies from cell to cell. It is conceivable that the same mitochondrion (or any part of it) may act alternatively as either a sink or a source of ROS. Various studies have been conducted on vitamin E and large number of positive data is suggestive that this compound has much potential in the managements of oxidative stress. Hence its availability, accessibility and its consumption should be brought into consideration of all individual. Health practicing individuals should be encouraged of its routine recommendation to their patients and smokers should be 
encouraged of it routine usage. In summary this study demonstrates the effects of smoking in term of raised HP which can eventually results in oxidative stress. Catalase and glutathione plays essential role in the detoxification mechanism of HP. However their individual roles at the molecular level are yet to be illustrated.

\section{Acknowledgements}

Shalamar Institutes of Health Sciences, Lahore, Pakistan for providing financial assistance in the form of research grant for the completion of this study.

\section{References}

[1] Veal E, Day A. (2011). Hydrogen peroxide as a signaling molecule. Antioxid Redox Signal. 1:147-151.

[2] Loo AEKiat, Wong YT, Ho R, Wasser M, Tiehua D, Wee T. et al. (2012) Effects of Hydrogen Peroxide on Wound Healing in Mice in Relation to Oxidative Damage. PLoS ONE. 11: e49215.

[3] Umit MS, Esra B, Serpil E, Cansin S, Omer K. (2011) Oxidative Stress in Asthma. World Allergy Organization. 4: 151-158.

[4] Fernando H. (2013) Oxidative Stress in Airway Diseases. Ann Am Thorac Soc. 10: S150-S157.

[5] Reinskje T, Thomas S, Ewa F, Jan-van B, Piet W, Antoon O. (2011) Hazardous Compounds in Tobacco Smoke. Int J Environ Res Public Health. 2: 613-628.

[6] Rockville. How Tobacco Smoke Causes Disease: (2010) The Biology and Behavioral Basis for Smoking-Attributable Disease. U.S. Department Of Health And Human Services. http://www.surgeongeneral.gov/library.

[7] http://www.compoundchem.com/2014/05/01/the-chemicalsin-cigarette-smoke-their-effects/

[8] Aydogan U, Durmaz E, Ercan CM, Eken A, Ulutas OK, et al. (2013) Smoking during pregnancy, DNA damage and ROS level. Arh Hig Rada Toksikol. 64: 35-46.

[9] Jiayuan Z, Philip KH. (2012) Concentration of Reactive Oxygen Species (ROS) in Mainstream and Side-stream Cigarette Smoke. Aerosol Science and Technology. 2: 191197.

[10] Zhao-Li C, Jian T, Jie Y, Zhen-Li Y, Xing-Hua L, Min J, et al. (2011) Vitamin E Modulates Cigarette Smoke Extract-induced Cell Apoptosis in Mouse Embryonic Cells. Int J Biol Sci. 7: 927-936.

[11] Corinne CW, Claudia PP, Peter G, Florence V, Gabriele S, et al. (2010) Hemoglobin can attenuate hydrogen peroxideInduced oxidative stress by acting as an antioxidative peroxidase. Antioxid Redox Signal. 2: 185-198.
[12] Pandey KB, Rizvi SI. (2011) Biomarkers of oxidative stress in red blood cells. Biomed Pap Med Fac Univ Palacky Olomouc Czech Repub. 2: 131-136.

[13] Mahapatra SK, Das S, Dey SK, Roy S. (2008) Smoking induced oxidative stress in serum and neutrophil of the university students. Al Ameen J Med Sci. 1: 20-31.

[14] Kosmider B, Messier EM, Chu HW, Mason RJ. (2011) Human Alveolar Epithelial Cell Injury Induced by Cigarette Smoke. PLoS ONE. 12: e26059.

[15] Betsuyaku $T$, Fuke S, Inomata $T$, Kaga K, Morikawa $T$, Odajima N. et al. (2013) Bronchiolar epithelial catalase is diminished in smokers with mild COPD. Eur Respir J. 42: 4253.

[16] Biju T, Amitha R, Sneha S, Rajendra P. (2013) A comparative evaluation of antioxidant enzymes and selenium in the serum of periodontitis patients with DM type 2. Contemporary clinical dentistry. 4: 176-180.

[17] Neal SG, Elysia M, Steve G, Richard JM, Brian JD. (2011) Lung glutathione adaptive responses to cigarette smoke exposure. Respiratory Research. 12: 133-142.

[18] Yousif YB, Sanaa S, Talal A, Shtywy A. (2012) Structure activity relationships regarding the antioxidant effects of the flavonoids on human erythrocytes. Natural Science. 4: 740 747.

[19] Mohamed J, Shing SW, Idris MH, Budin SB, Zainalabidin S. (2013). The protective effect of aqueous extracts of roselle against red blood cell membrane oxidative stress in rats with streptozotocin-induced diabetes. Clinics. 68: 1358-1363.

[20] Abbas M, Ali R. (2013) Erythrocyte osmotic fragility test revealed protective effects of supplementation with saffron and cinnamon on the Red Blood Cell membrane. Asian J. exp. biol. sci. 2: 322-326.

[21] Ergueder, Imge B., et al. (2009) The effects of cigarette smoking on serum oxidant status, and cholesterol, homocysteine, folic acid, copper, and zinc levels in university students. Turkish Journal of Medical Sciences. 4: 513-517.

[22] Padmavathi, Pannuru, Vaddi Damodara Reddy, and Nallanchakravarthula Varadacharyulu. (2009) Influence of chronic cigarette smoking on serum biochemical profile in male human volunteers. Journal of Health science. 2: 265-270.

[23] Cano, Marisol, et al. (2010) Cigarette smoking, oxidative stress, the anti-oxidant response through $\mathrm{Nrf} 2$ signaling, and Age-related Macular Degeneration. Vision research. 7: 652664 .

[24] Rao, P. S. S., Ande, A., Sinha, N., Kumar, A., \& Kumar, S. (2016). Effects of cigarette smoke condensate on oxidative stress, apoptotic cell death, and HIV replication in human monocytic cells. PloS one. 5: e 0155791. 